\title{
Action model of infall into the Virgo cluster
}

\author{
R. Brent Tully ${ }^{1,2}$ and Roya Mohayaee ${ }^{2}$ \\ ${ }^{1}$ Institute for Astronomy, University of Hawaii, Honolulu, HI 96822, USA email: \\ tully@ifa.hawaii.edu \\ ${ }^{2}$ Observatoire de la Côte d'Azur, 06304 Nice, France email: roya@obs-nice.fr
}

\begin{abstract}
The observed infall of galaxies into the Virgo cluster puts strong constraints on the mass of the cluster. A non-parametric fully non-linear description of the infall can be made with orbit reconstructions based on Numerical Action Methods. The mass of the cluster is determined to be $1.2 \times 10^{15} M_{\odot}$. The mass-to-light ratio for the cluster is found to be seven times higher than the mean ratio found across the region within $V=3000 \mathrm{~km} / \mathrm{s}$.
\end{abstract}

\section{Introduction}

The general problem we will consider is the reconstruction of orbits that galaxies might have followed to arrive at their currently observed positions on the sky and in redshift. On this occasion we will focus on the particularly interesting circumstances associated with infall into the Virgo cluster. The program involves three distinct components.

First, we require as complete a map as possible of the angular positions and velocities of galaxies. It will be assumed that there is some correlation between the distribution of galaxies in the catalog and the actual distribution of mass, which is the parameter that really interests us. However it is to be anticipated from the outset that the relationship between what we see - galaxies of various types - and the distribution of mass may be complex (Tully 2003, 2004). It is also to be appreciated that large scale tides may be dynamically significant so one needs a map of the distribution of galaxies that extends well beyond the region of immediate focus. In the present instance, our interest is in the environs of the Virgo cluster at a distance of $16.8 \mathrm{Mpc}$. Our map of the distribution of galaxies is based on a sample of 3151 galaxies within $\sim 40 \mathrm{Mpc}$. The sample is complete to $0.1 L^{\star}$ within $25 \mathrm{Mpc}$ at high galactic latitudes. Selection function corrections are made as a function of distance and 'fake' sources are added at low latitudes to account for missing sources in the zone of obscuration.

The second critical component is a catalog of accurate distances to galaxies. Distance measures, $d$, allow a separation of observed velocities, $V_{\text {obs }}$, and peculiar velocities, $V_{\text {pec }}$, since $V_{\text {pec }}=V_{\text {obs }}-\mathrm{H}_{0} d$. Here, $\mathrm{H}_{0}$ is the Hubble constant which is taken to be 80 $\mathrm{km} / \mathrm{s} / \mathrm{Mpc}$. For purposes of separating $V_{\text {pec }}$ from $V_{\text {obs }}$ it is only required that distances and the Hubble constant be on the same scale; that is, with averaging that takes into account large scale flows: $\mathrm{H}_{0}=<V_{\text {obs }} / d>$. If distances are, say, systematically measured too large then a self-consistent value of $\mathrm{H}_{0}$ will be too low. Derived values of $V_{\text {pec }}$ are independent of the distance scale zero-point. Another factor with regard to distances to appreciate is that errors are a percentage of the distance, $10 \%$ to $20 \%$ depending on the methodology, with the consequence that errors in the derived $V_{\text {pec }}$ grow linearly with distance. At the Virgo cluster a $10 \%$ error corresponds to $\pm 140 \mathrm{~km} / \mathrm{s}$ in $V_{\text {pec }}$. This uncertainty is tolerable for the Virgo infall problem but for more distant clusters the situation would be unsatisfactory. The distance catalog itself is a synthesis of our own observations (Pierce and Tully, in preparation) that exploit the luminosity-linewidth 
method (Tully and Fisher 1977) and material from the literature. That literature material provides distances from a variety of methodologies: luminosity-linewidth (Mathewson et al. 1992, Lu et al. 1993, Tully and Pierce 2000), Cepheid (Freedman et al. 2001), Tip of the Giant Branch (Karachentsev et al. 2003), Planetary Nebula Luminosity Function (Jacoby et al. 1990), and Surface Brightness Fluctuation (Tonry et al. 2001). This latter important source is distinguished separately in the ensuing discussion. In total we have distances to almost 900 galaxies from the luminosity-linewidth and other assorted methods and 292 distances from the Surface Brightness Fluctuation method.

The third ingredient is the theoretical machinery to convert information on the amplitude of the peculiar velocities of galaxies into a map of the mass distribution. In linear theory there is a direct relation between $V_{\text {pec }}$ and matter density fluctuations. The relationship is more complex in the vicinity of collapsed structures, such as the Virgo cluster. We make use of Numerical Action Methods which we have referred to as 'Least Action'. The numerical techniques have been discussed by Peebles $(1989,1995)$, Shaya et al. (1995), and Phelps (2002). The procedure allows for orbit reconstructions in highly nonlinear regimes, though ambiguity arises if the orbits are complex, especially if dynamical friction or orbital energy exchange is important. These are not serious issues for the Virgo infall problem.

\section{Numerical Action Methods}

The 'Action' is defined as the integral along paths of the Lagrangian, the kinetic minus the potential energy. The equations of motion for particle orbits satisfy minima of the derivatives of the action with respect to position and momentum. Orbits of particles that represent saddle points of the action and agree with boundary conditions are also physically plausible.

Orbits are described by the 6 elements of phase space $\left(\alpha_{i}, \delta_{i}, d_{i}, V_{i}^{\alpha}, V_{i}^{\delta}, V_{i}^{r}\right)$ under the influence of the ensemble of mass elements, $m_{i}$, and a time frame. In the ensuing discussion we will assume a flat universe with the matter density as a fraction of the closure density $\Omega_{m}=0.21$, the vacuum energy fractional density $\Omega_{\Lambda}=0.79$, and $\mathrm{H}_{0}=80$ $\mathrm{km} / \mathrm{s} / \mathrm{Mpc}$. This choice of parameters sets the clock. We solve a mixed boundary value problem constrained by the 3 well-known elements of phase space today $\left(\alpha_{i}, \delta_{i}, V_{i}^{r}\right)$ and the theoretically motivated condition that initially the 3 components of velocity were negligible; i.e. taken to be zero. Then given mass assignments to the components of our galaxy catalog, orbits can be constructed.

The observed luminosities of the components of the catalog, $\ell_{i}$, provide a guide for the mass assignments. Given $m_{i}=\ell_{i}\left(m_{i} / \ell_{i}\right)$, the uncertainties in $m_{i}$ can be transformed into uncertainties in $m_{i} / \ell_{i}$. Hence, with specification of mass-to-light values we can constrain plausible orbits. There would be too many degrees of freedom if all $m_{i} / \ell_{i}$ were allowed to be different so, to begin, we go to the other limit and assume all objects have the same value, $M / L$.

Given a choice of $M / L$ within the context of a specified cosmology we can constrain plausible orbits, but how can discrimination be made between alternatives? It is here that we make use of distance measurements. The orbits that have been defined are constrained as boundary conditions to have specified positions on the sky and radial velocities at the current epoch. The end point distances are an output of the model. For a substantial fraction of the objects we have observed distances. These cases can be used to evaluate the quality of the model through a $\chi^{2}$ estimator that compares differences between model and observed distances (the comparison is done in the logarithms using distance moduli). 


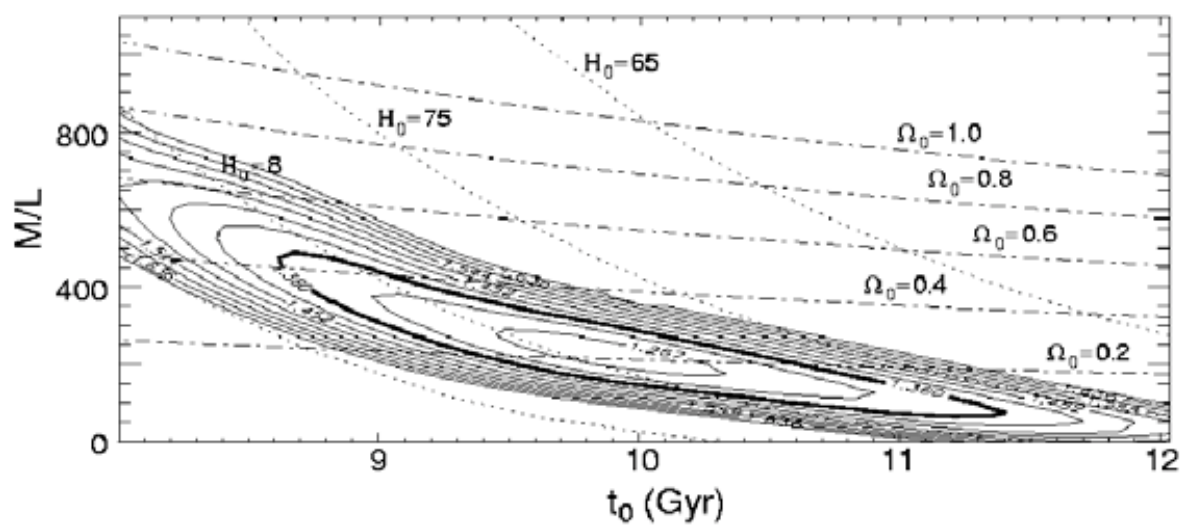

Figure 1. Contours of constant $\chi^{2}$ from an Action analysis of a catalog restricted to $V<3000$ $\mathrm{km} / \mathrm{s}$. In this case, $\Omega_{\Lambda}=0$ is assumed. The heavy contour represents the $2 \sigma$ confidence level.

Figure 1 provides an example of $\chi^{2}$ contours in the domain of $M / L$ vs. $t_{0}$, the age of the universe. The analysis presented here assumed $\Omega_{\Lambda}=0$ and the distance scale zero point was $10 \%$ above our current preference. These differences particularly affect the time domain and, at the $10 \%$ level, the $M / L$ values but do not affect the validity of the $\Omega_{m}$ estimates.

It is to be emphasized what one should not and what one should take seriously with the Action models. One should not take seriously the specific orbit reconstructions. The orbits that are derived are physical but not unique. The uniqueness problem becomes important for large-scale reconstruction where a different method which guarantees uniqueness, can be employed (Frisch et al. 2002). One should take seriously the constraints on mass. A thought experiment can make this point clear. Suppose we consider two cases of interaction between a test particle and a substantial mass where the difference between the two cases is simply the mass of the major attractor. Suppose, as in the real world, we can measure the line-of-sight velocity of the test particle with high accuracy and measure the separation between the test particle and attractor with some error. We want to estimate the mass of the attractor. Admitting that the line-of-sight velocity is only a component of the true velocity we only have a statistical test. However it should be clear that the measure of the separation between particles provides a measure of the mass. For a specified infall velocity, if the attractor has more mass then the test particle has to be farther away. The Action models are responding to this effect. If there is generally more mass, then for fixed velocities and within a specified cosmological model, objects are farther apart. Our distance observations are distinguishing between the mass possibilities.

We are careful to demand that the cosmological model be specified because, as is clear from Fig. 1, there is a trade off between mass and time. A model with more mass and less time can duplicate a model with less mass and more time. Essentially the effect of $\Omega_{\Lambda}>0$ is to change the clock.

\section{Virgo infall}

Probably of all the problems we could consider with the Action machinery, the simplest application is in connection with infall into the Virgo cluster. Here we are giving consideration to the first approach infall of essentially 'test particles' toward a dominant mass. Near to the cluster, the radial motions toward the cluster (reaching $1500 \mathrm{~km} / \mathrm{s}$ ) 
are much larger than any transverse motions $(\sim 100 \mathrm{~km} / \mathrm{s})$. Since the orbits are essentially radial, on first approach, and have not yet reached the cluster, there are no serious concerns about orbital energy exchange or dynamical friction. The interactions involve only small perturbations on a two-body encounter.

We begin with Action model parameters that provide a reasonable $\chi^{2}$ fit to distance measures throughout the region with $V<3000 \mathrm{~km} / \mathrm{s}$. Initially, a single value of $M / L$ is taken for all components. Fig. 1 provides an example of a good solution. In the present study, we assume a flat cosmological model with $\Omega_{m} h^{2}=0.135$ (where $h=\mathrm{H}_{0} / 100$ ) consistent with microwave background measurements (Spergel et al. 2003) but take the slightly nonstandard values $h=0.80$ and $\Omega_{m}=0.21$.

As we have reported earlier (Shaya et al. 1995), good fits to the ensemble of the data within $V=3000 \mathrm{~km} / \mathrm{s}$ with a single $M / L$ value assigned to all components requires a low $M / L \sim 200 M_{\odot} / L_{\odot}$. However a part of the data is poorly fit with this solution. This model implies a sufficiently modest mass for the Virgo cluster that it cannot generate the large infall velocities that are observed.

The so-called 'triple-value' region (Tonry and Davis 1981) around a cluster provides a signature of infall that depends on the mass of the cluster. Theoretical models demonstrate the properties of infall in 3-dimensions (Bertschinger 1985). The structure is simple in the volume within the zone of first collapse into the radius of a caustic which is the outer extent of orbits of objects that have passed through the cluster once and reached second turnaround. Within this zone between outer (first turnaround) and inner (second turnaround) radii the infall pattern translates to the now familiar 'triple-value' wave, whereby there are three locations that give the same velocity. In such instances, one of the locations lies outside the first turnaround - or zero velocity surface for the cluster - and the other two are within the collapse region. There are two infall locations that give the same velocity because of a line-of-sight geometrical effect. Since the motions represent infall toward the cluster, objects on the front side of the cluster have higher velocities than the cluster mean and objects on the back side of the cluster have lower velocities than the cluster mean.

There are two key points to understand in connection with the present study. The first is the obvious point that the amplitude of the triple-value wave depends directly on the mass of the cluster. Hence, if one is convinced that an object is within the infall region (rather than at the location outside the zero-velocity surface) then the mass of the cluster needs to be sufficient to explain the velocity of that object. In many cases in which the velocities are similar to that of the cluster the demand will not be too great. The challenge is to explain the extreme cases.

The second point to appreciate is that the Virgo cluster is near enough that we generally can unambiguously determine whether a target is within or outside the infall region. We may not be able to distinguish between the two infall options but that is of minor consequence. The essential point is that if the object is within the infall region then that object in itself puts a minimal demand on the mass interior to its position within the infall domain.

The general domain of the Virgo infall region is demonstrated in Figure 2. It is observationally determined that the outer radius of first turnaround is about $28^{\circ}$ from the center of the cluster (a radius of $8 \mathrm{Mpc}$ ) while the caustic of the second turnaround is at about $6^{\circ}(1.8 \mathrm{Mpc})$. In Fig. 2, all the objects that are highlighted (plus others), except for the object 102, are within the Virgo cluster zero-velocity surface. It is seen that many of these objects are to the left of the cluster in this plot. These objects lie in the 'Virgo Southern Extension' which is a cloud of galaxies impinging on the cluster at the present epoch. 


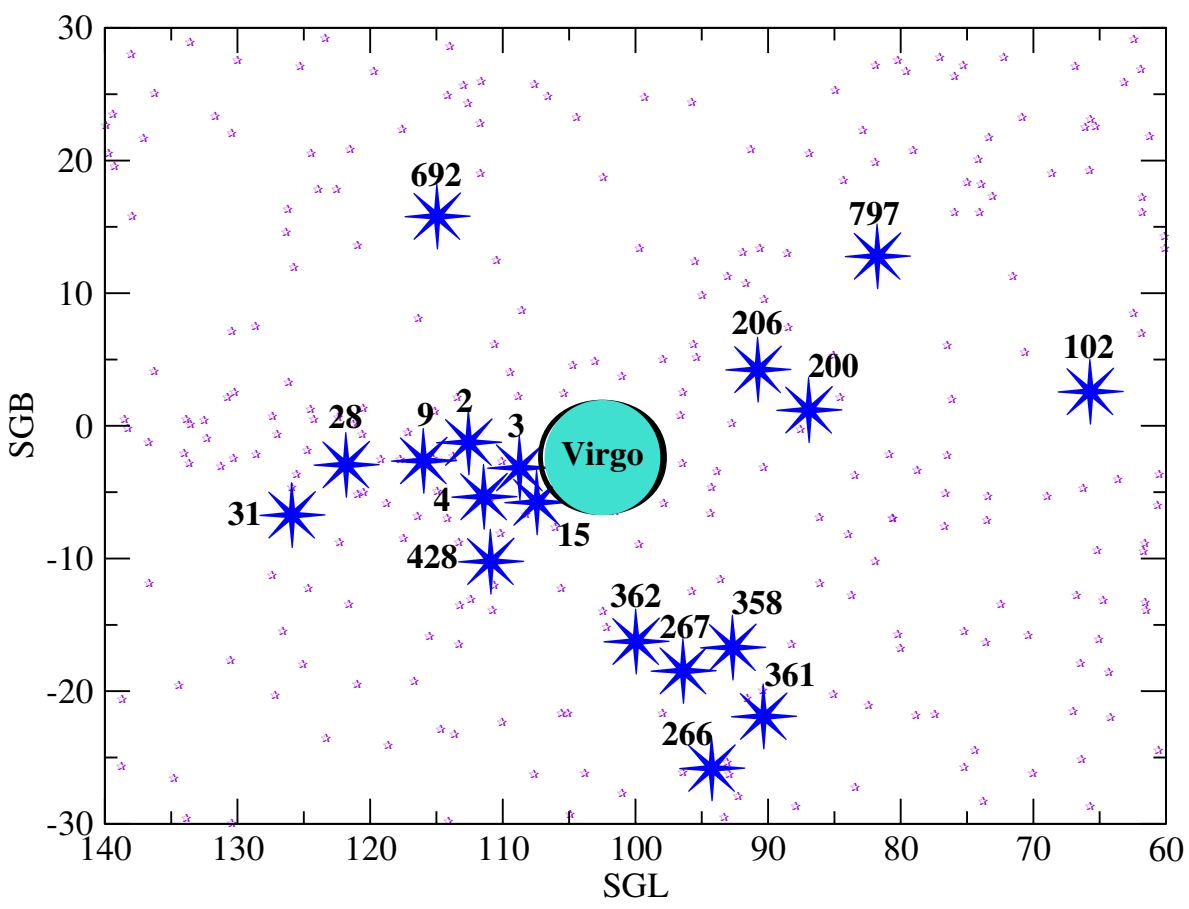

Figure 2. Projection on the sky of the Virgo cluster and surrounding region. The various symbols indicate the positions of objects projected in the vicinity of the cluster and those noted by large stars and numbered are particularly 'interesting' from the standpoint of the Virgo infall problem. The infall region extends to $\sim 28^{\circ}$ around the cluster, encompassing all but the object labelled 102 on this plot.

The objects highlighted in Fig. 2 have received special attention because they have good distance measures and many of them provide strong constraints on the cluster mass. An example is presented in Figure 3. The two panels illustrate the triple-value wave in the direction of a specific group of galaxies (object 4 in Fig. 2: the 11-4 Group in Tully 1988) with the only difference between the two cases being the Virgo cluster mass assignment. In both cases, the Action model was solved assuming $M / L=125 M_{\odot} / L_{\odot}$ as a baseline. In the case shown on the right, however, the mass of the clusters dominated by early type systems (for present purposes that means the Virgo cluster) was augmented by 7 to $M / L=875 M_{\odot} / L_{\odot}$. The mass of the cluster was increased from $1.6 \times 10^{14} M_{\odot}$ (left panel) to $1.2 \times 10^{15} M_{\odot}$ (right panel). The measured distance and velocity of the 11-4 Group is indicated by the point with error bars. The group of stars indicate the distance and velocity of the Virgo cluster as modelled by a series of Action trials. The ' $\mathrm{x}$ ' indicate distances and velocities determined for the 11-4 Group with the Action trials. The points that describe waves rising from zero distance and velocity to $40-45 \mathrm{Mpc}$ at $3000 \mathrm{~km} / \mathrm{s}$ are the orbital end points of test particles scattered along the line-of-sight of the 11-4 Group.

There is a clear difference between the two figures in the amplitude of the triple-value wave in the vicinity of the Virgo cluster. This difference is a consequence of the different cluster mass assignments. The wave defined by the test particles shows where objects could lie in the context of the specified model. In the right panel of Fig. 3, the data point representing the 11-4 Group lies on the triple-value wave, hence the model accommodates this datum. In the left panel, however, the same data point lies well away from the much 

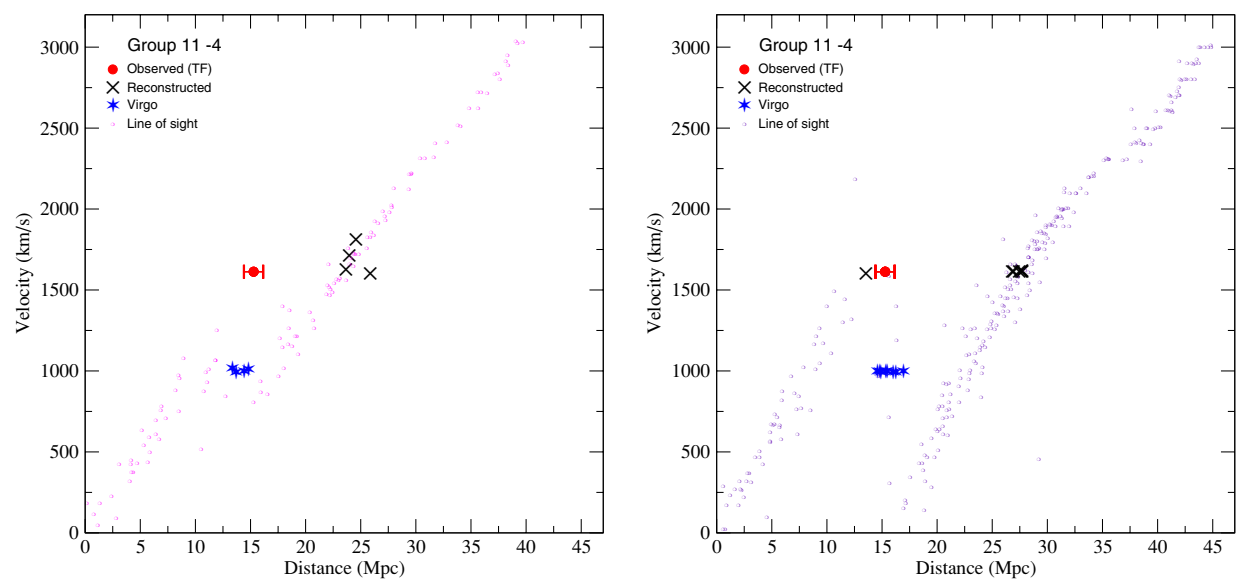

Figure 3. The left plot is the (limited) triple-value wave associated with the line-of-sight directed at the infall group 11-4 in action models with $M / L=125 M_{\odot} / L_{\odot}$ for all objects. In this case the mass of the Virgo cluster is $1.7 \times 10^{14} M_{\odot}$. The mean Virgo cluster distance and velocity given by several Action trials is indicated by the stars. The locations found for the 11-4 group in different trials with the Action models are given by the ' $x$ '. The point with error bars indicate the observed position and velocity of the group. The right plot is the (much more extensive) triple-value wave along the same line of sight toward the 11-4 group generated by action models still with $M / L=125 M_{\odot} / L_{\odot}$ for most objects but now with the mass of the Virgo cluster (and other E/S0 groups in the catalog) amplified by a factor 7 . The Virgo cluster is now given a mass of $1.2 \times 10^{15} M_{\odot}$. Symbols have the same meaning as in the left panel.

more modest triple-value wave. We are forced to conclude that either the data point is unreliable or the model shown in this figure is bad. The ' $x$ ' symbols that illustrate the positions found for the 11-4 Group in the Action models reinforce the point. In the left panel, the Action models put the group in the vicinity of $25 \mathrm{Mpc}$, in expansion away from the cluster, rather than at it's measured distance of $15.3 \pm 0.9 \mathrm{Mpc}$. In the right panel, the Action models frequently put the group at a similar background position but on an occasion a saddle point of the action was chosen in good agreement with the observed distance. Clearly, in a computation of the $\chi^{2}$ evaluator of the distance agreement this latter case is strongly favored. The important point to be made is that in order for an infall solution to be available for the 11-4 Group the mass of the cluster has to be high enough to generate a triple-value wave with an amplitude that catches the velocity observed for the group.

\section{Conclusions}

Similar analyses can be made of dozens of other lines-of-sight through the infall domain around the Virgo cluster. If there is a distance measurement that confirms that a target is inside the zero-velocity surface around the cluster then the line-of-sight component of its velocity puts a constraint on the cluster mass. In each case, the cluster mass must be at least enough to provide an Action infall solution.

We determine that a minimum but sufficient Virgo cluster mass for the models is $1.2 \times 10^{15} M_{\odot}$. This mass is $50 \%$ larger than the mass determined from application of the virial theorem (Tully and Shaya 1984). However, the virial radius for the cluster is $\sim 0.8 \mathrm{Mpc}$ while the mass measured by infall is on a scale $\geqslant 1.8 \mathrm{Mpc}$. The infall analysis, 
which is based on very simple physics, gives an estimate of the global mass of the cluster. It can be anticipated that this mass will exceed the masses determined over more limited scales by gravitational lensing, X-ray, or virial studies.

Unfortunately, the analysis is not easily duplicated on other clusters, at least not so cleanly. The Virgo cluster is the only environment near enough that distance measurements distinguish between infall and expansion regimes, and massive enough to have an extensive, well populated infall domain. It provides a single good case. What we find, though, is that whereas overall the Action models find preference for $M / L \sim 125 M_{\odot} / L_{\odot}$ and $\Omega_{m} \sim 0.2$, in the Virgo cluster we need $M / L \sim 900 M_{\odot} / L_{\odot}$.

\section{Acknowledgements}

This research is being carried out in close collaboration with Jim Peebles, Steven Phelps, and Ed Shaya. Support for RBT is provided by JPL Contract 1243647. RM is supported by the European Union Marie Curie Fellowship HPMF-CT-2002-01532.

\section{References}

Bertschinger, E. 1985, ApJ, 58, 39

Freedman, W.L. et al. 2001, ApJ, 553, 47

Frisch U., Matarrese S., Mohayaee R. and Sobolevskii A. 2002, Nature, 417, 260

Jacoby, G.H., Ciardullo, R., Ford, H.C. 1990, ApJ, 356, 332

Karachentsev, I.D. et al. 2003, A\&A, 398, 493

Lu, N.Y. et al. 1993, ApJS, 88, 383

Mathewson, D.S., Ford, V.L., Buchhorn, M. 1992, ApJS, 81, 413

Peebles, P.J.E. 1989, ApJ, 344, L53

Peebles, P.J.E. 1995, ApJ, 449, 52

Phelps, S.D. 2002, ApJ, 575, 1

Shaya, E.J., Peebles, P.J.E., Tully, R.B. 1995, ApJ, 454, 15

Spergel D.N. et al. 2003, ApJ, 148, 175

Tonry, J.L., Davis, M. 1981, ApJ, 246, 680

Tonry, J.L. et al. 2001, ApJ, 546, 681

Tully, R.B. 2003, astro-ph/0312441

Tully, R.B. 2004, astro-ph/0402516

Tully, R.B. 1988, Nearby Galaxies Catalog, Cambridge University Press

Tully, R.B., Fisher, J.R. 1977, A\&A, 54, 661

Tully, R.B., Pierce, M.J. 2000, ApJ, 533, 744

Tully, R.B., Shaya, E.J. 1984, ApJ, 281, 31 